\title{
Canals beyond Mars: Beam depolarization in radio continuum maps of the warm ISM
}

\author{
M. Haverkorn ${ }^{1,2}$ and F. Heitsch ${ }^{3,4,5}$ \\ 1 Sterrewacht Leiden PO Box 9513, 2300 RA Leiden, The Netherlands \\ 2 Harvard-Smithsonian Center for Astrophysics, 60 Garden Street MS-67, Cambridge, MA, 02138, USA \\ 3 Max-Planck-Institut für Astronomie, Königstuhl 17, 69117 Heidelberg, Germany \\ ${ }^{4}$ University of Wisconsin-Madison, 475 N Charter St, Madison, WI 53706, USA \\ 5 Institut für Astronomie und Astrophysik, Universität München, Scheinerstr. 1, 81679 München, Germany \\ e-mail: heitsch@usm.uni-muenchen.de
}

Received 5 October 2003 / Accepted 8 March 2004

\begin{abstract}
Multi-frequency radio polarimetric observations of the diffuse Galactic synchrotron background enable us to study the structure of the diffuse ionized gas via rotation measure maps. However, depolarization will introduce artifacts in the resulting rotation measure (RM), most notably in the form of narrow, elongated "depolarization canals". We use numerical models of a non-emitting Faraday rotating medium to study the $R M$ distribution needed to create depolarization canals by depolarization due to a finite beam width, and to estimate the influence of this depolarization mechanism on the determination of $R M$. We argue that the depolarization canals indeed can be caused by beam depolarization, which in turn is a natural consequence when observing a turbulent medium with limited resolution. Furthermore, we estimate that beam depolarization can induce an additional error of about $20 \%$ in $R M$ determinations, and considerably less in regions that are not affected by depolarization canals.
\end{abstract}

Key words. magnetohydrodynamics - magnetic fields - polarization - turbulence - ISM: magnetic fields radio continuum: ISM

\section{Introduction}

The Galactic magnetic field and turbulence play a major rôle in structuring the Galactic disk. The recognition of the dynamical importance of turbulence in the interstellar medium (ISM) (e.g., Armstrong et al. 1995) allowed our picture of the ISM to evolve from a simple three-phase gas to a highly complex medium. Turbulence is believed to play a crucial rôle in molecular cloud and star formation (see e.g., Larson 1981; Mac Low $\&$ Klessen 2003), however, its origin is widely debated. On a larger scale turbulence leads to chemical mixing (de Avillez \& Mac Low 2002) and contributes to the vertical pressure balance in the Galactic disk and to the heating of the ISM (Minter \& Balser 1998).

Magnetic fields are tightly linked to the turbulent nature of the ISM. Their large-scale components help confine cosmic rays to the Galaxy, and thus affect chemical processes in molecular clouds. They probably contribute to generating turbulence in the disk (Sellwood \& Balbus 1999) and definitely affect the vertical disk structure. The ratio between mass and magnetic flux in the ISM is an essential piece of information necessary

Send offprint requests to: $\mathrm{M}$. Haverkorn, e-mail: mhaverkorn@cfa.harvard.edu to understand the rôle of magnetic fields in molecular cloud and eventually star formation (see e.g., Crutcher 1999).

Density and magnetic field structure in the ISM can be probed using multi-frequency radio polarimetry to study Faraday rotation ${ }^{1}$ in the magneto-ionic medium. Polarized extragalactic point sources and pulsars have been used to determine rotation measures $(R M)$, but these give only measurements at certain lines of sight. Observing the diffuse Galactic synchrotron background instead yields structure in polarized emission on many scales, along many contiguous lines of sight. However, depolarization complicates the interpretation of the resulting $R M$ maps. Depolarization occurs if the telescope beam is larger than the scale of structure in polarization (beam depolarization), along the line of sight if the radiation is emitted and Faraday rotated largely in the same medium (depth depolarization or internal Faraday dispersion), or if the frequency

\footnotetext{
Faraday rotation is the rotation of the plane of linear polarization of radiation when it propagates through a magneto-ionic medium, due to the birefringence of the medium to circularly polarized radiation. The rotation of the polarization plane through the medium is $\Delta \phi=R M \lambda^{2}$, with rotation measure $R M=0.81 \int B_{\|} n_{\mathrm{e}} \mathrm{d} s$, where $B_{\|}$ is the component of the magnetic field parallel to the line of sight in $\mu \mathrm{G}, n_{\mathrm{e}}$ is the thermal electron density in $\mathrm{cm}^{-3}$ and $\mathrm{d} s$ is the path length in pc.
} 
bandwidth is so large that the polarization angle changes significantly within the band (bandwidth depolarization), see e.g., Burn (1966) or Sokoloff et al. (1998). In polarization observations of the Galactic synchrotron background, often it is difficult if not impossible to estimate the separate contributions of beam and depth depolarization.

Depolarized regions often exhibit canal-like features (e.g., Duncan et al. 1997, 1999; Gray et al. 1999; Uyanıker et al. 1999; Haverkorn et al. 2000; Gaensler et al. 2001). Both beam and depth depolarization can cause canals, but require a different underlying $R M$ distribution. The goal of this paper is to determine if the observed depolarization canals can be due to beam depolarization, and how beam depolarization affects the computed $R M$. To this end, we use numerical models of the warm magneto-ionic ISM compared to observations of the diffuse Galactic synchrotron background. The models are irradiated with uniform synchrotron background emission, which is Faraday rotated in the modeled medium. Then, the polarized radiation is smoothed to simulate a finite telescope beam, whereafter $R M \mathrm{~s}$ are computed from the smoothed emission maps. The structure in polarized intensity and $R M$ emerging from the smoothed modeled radiation field is then compared to the observations. This allows us to simulate the effect that beam depolarization has on polarimetric observations of the ionized ISM.

This work was motivated by the observations briefly presented in Sect. 2. In Sect. 3 we describe the numerical models, the addition of the synchrotron background and the simulation of beam depolarization. Section 4 discusses the signature of beam depolarization in the maps of polarized intensity, while in Sect. 5 the effect of beam depolarization on $R M$ is studied.

\section{The observations}

The observations were made with the Westerbork Synthesis Radio Telescope (WSRT) in five frequency bands centered on $341,349,355,365$, and $375 \mathrm{MHz}$, each with a bandwidth of $5 \mathrm{MHz}$. The field discussed here is centered at $(l, b)=$ $\left(161^{\circ}, 16^{\circ}\right)$ and about 50 square degrees in size, and has a resolution of about $4^{\prime}$. All four Stokes parameters $I, Q, U$ and $V$ are measured, from which polarized intensity $P$ and polarization angle $\phi$ were determined as

$$
\begin{aligned}
P & =\sqrt{Q^{2}+U^{2}} \\
\phi & =\frac{1}{2} \arctan \frac{U}{Q}
\end{aligned}
$$

The instrumental linear polarization is $\lesssim 1 \%$. For a detailed description of this field see Haverkorn et al. (2003a).

No signal was detected in total intensity $I$. As the interferometer is only sensitive to scales smaller than approximately a degree, the non-detection of $I$ means that $I$ does not exhibit any structure on scales smaller than $\sim 1^{\circ}$, so that the observed total synchrotron power must be very uniform. On the other hand, abundant structure in linearly polarized emission was detected, both in $P$ (see Fig. 1) and in $\phi$. This indicates that the small-scale structure in polarization is caused by Faraday rotation and depolarization. Ubiquitously present in the field are one-beam wide "canals" of beam depolarization, which we will

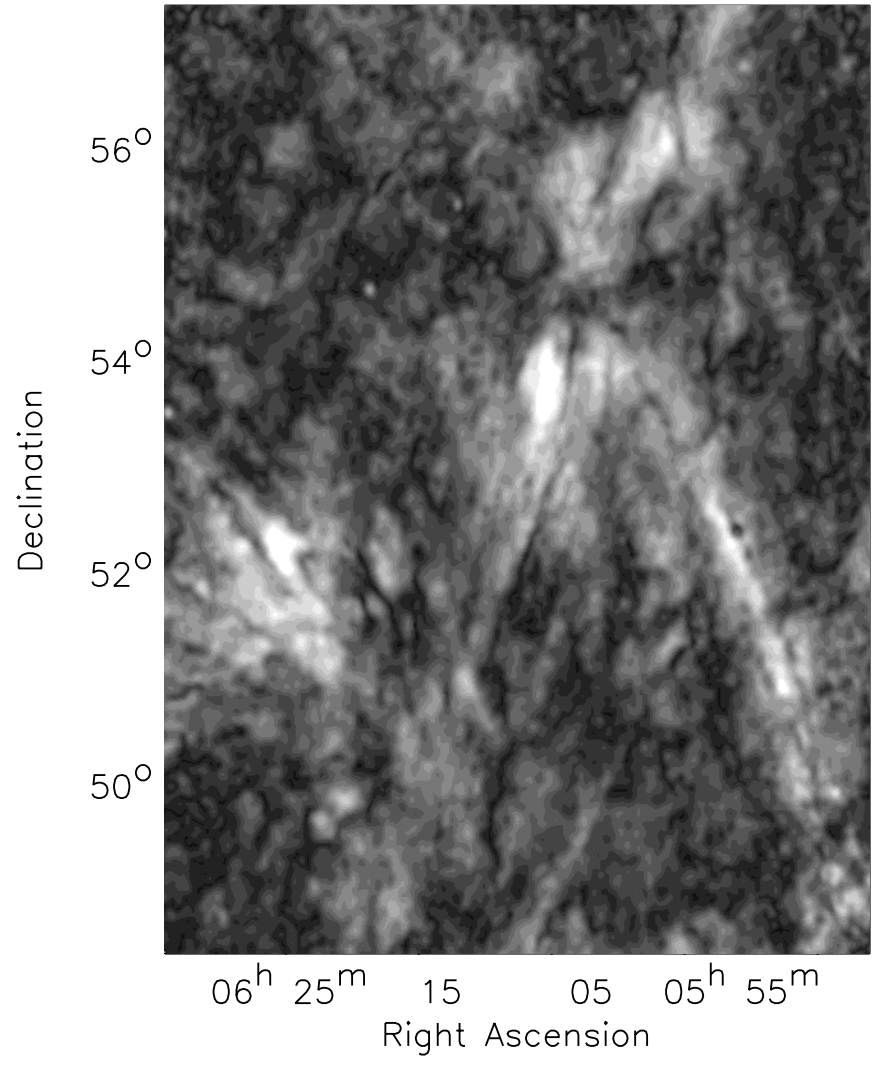

Fig. 1. Observed polarized intensity at $349 \mathrm{MHz}$ in a field centered at $(l, b)=\left(161^{\circ}, 16^{\circ}\right)$. High intensity is shown white, up to a maximum of $\sim 13 \mathrm{~K}$.

discuss in Sect. 4. Missing large-scale structure in $P$ probably does not play a large rôle in this field (Haverkorn et al. 2003a, 2004b).

$R M \mathrm{~s}$ were computed from linear fits of $\phi$ against $\lambda^{2}$. For about $70 \%$ of the beams with an average polarized intensity $\langle P\rangle>5 S / N$, reliable $R M$ 's (with reduced $\chi^{2}<2$ ) could be determined. This is about $28 \%$ of all data. Figure 2 shows a grey scale map of $R M$, where in each beam a $R M$ value is plotted, regardless of the quality of fit of the $\phi\left(\lambda^{2}\right)$-relation. In general, the positions showing anomalous $R M$ with rapidly changing magnitude and sign are positions where noise dominates, so that reliable $R M$ determination is not possible, see Sect. 4 .

Contributions to the $R M$ are made along the line of sight at positions with a non-zero electron density and magnetic field. This is the case in the warm and hot ISM components. The filling factor in the warm ISM is approximately $20 \%$ (Reynolds 1991), while the electron density in the hot ISM is so low that its $R M$ contribution can be neglected for our purposes. Furthermore, polarized emission is increasingly depolarized when emitted at greater distances. Haverkorn et al. (2004a) estimate a distance of $\sim 600 \mathrm{pc}$ between observer and the "polarization horizon", defined as the point beyond which less than about a third of the polarized emission is not depolarized while propagating through the medium. 


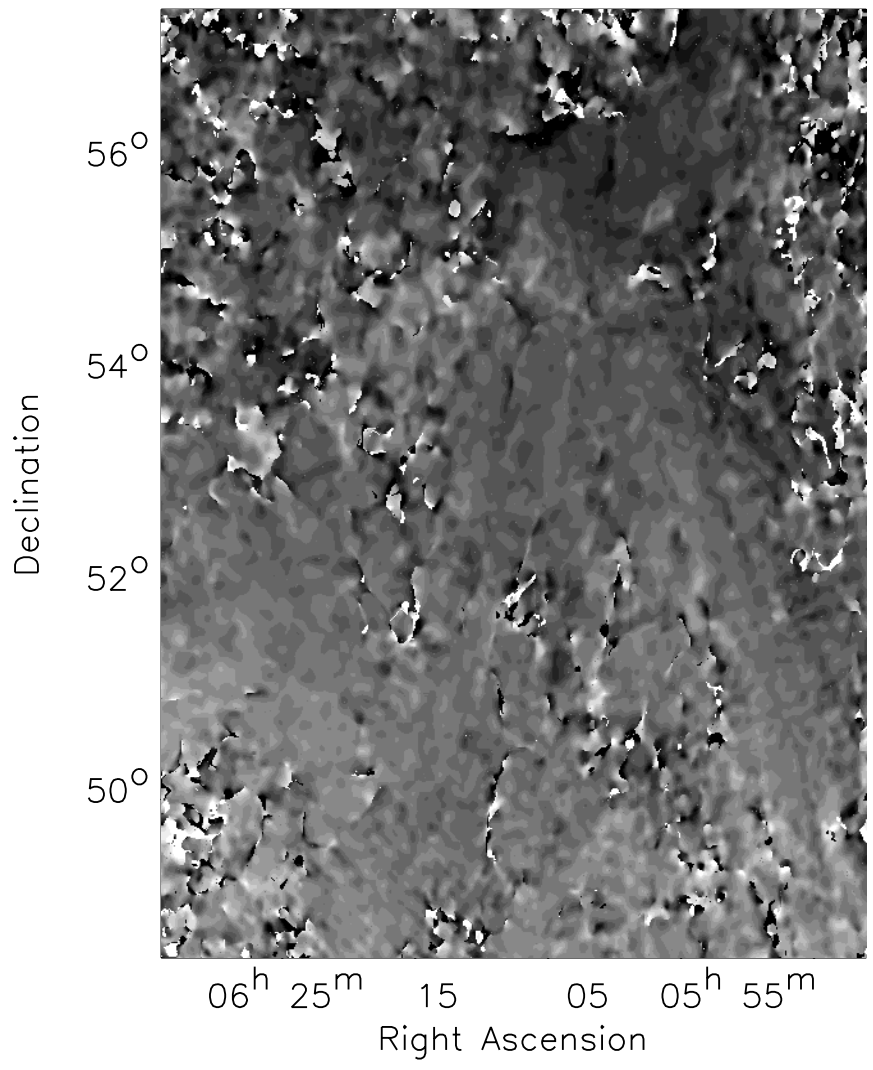

Fig. 2. Map of $R M$ in the observed field. The grey scale runs from -15 to $15 \mathrm{rad} \mathrm{m}^{-2}$. The map shows an $R M$ value at every position, regardless of the quality of the linear fit of $\phi$ against $\lambda^{2}$.

\section{Numerical models}

To estimate the effect of beam depolarization on the $R M$ determinations, we generate artificial maps of polarized intensity at five wavelengths from numerical models of a turbulent magnetized ISM.

\subsection{Domain considerations}

Ideally, the numerical models should resemble the observational domain (i.e., the ratio between the length of the line of sight and the extent in the plane of sky) while retaining a resolution in the plane of sky comparable to the observations. At an estimated polarization horizon of $600 \mathrm{pc}$, the spatial extent of the $7^{\circ} \times 9^{\circ}$ field of observation is about $75 \mathrm{pc}$. The observational resolution of $4^{\prime}$ would correspond to approximately $120 \times 120$ resolution elements in the plane of sky.

However, for the numerical models, this estimated requirement is too optimistic for two reasons. First, in order to investigate beam depolarization, we need substructure within one beam (i.e. resolution element). Second, any magnetohydrodynamics (MHD) code suffers from numerical dissipation at the smallest scales, thus introducing a lower limit for physically reasonable structures in the simulations (between 2 to 4 resolution elements in our case). Both requirements taken together lead to a desirable resolution of $\sim 500 \times 500$ elements in the plane of sky for the simulations, resulting in a domain size of
Table 1. Parameters of the numerical models used: $N_{x}, N_{y}$ and $N_{z}$ are the model dimensions in resolution elements and $L_{\mathrm{los}}$ is the length of the line of sight in parsecs. The initial magnetic field $B_{\|}$is oriented along the line of sight $z$ and $n_{\mathrm{e}}$ is the initial uniform electron density. $\triangle R M$ is the width $\sigma$ of the Gaussian distribution of $R M$, scaled via $L$.

\begin{tabular}{cccccc}
\hline \hline name & $N_{x} \times N_{y} \times N_{z}$ & $\begin{array}{c}L_{\mathrm{los}} \\
{[\mathrm{pc}]}\end{array}$ & $\begin{array}{c}B_{\|} \\
{[\mu \mathrm{G}]}\end{array}$ & $\begin{array}{c}n_{\mathrm{e}} \\
{\left[\mathrm{cm}^{-3}\right]}\end{array}$ & $\begin{array}{c}\Delta R M \\
{\left[\mathrm{rad} \mathrm{m}^{-2}\right]}\end{array}$ \\
\hline$B 1$ & $512 \times 512 \times 4096$ & 100 & 2.5 & 0.1 & 3 \\
$B 2$ & $512 \times 512 \times 4096$ & 200 & 2.5 & 0.1 & 6 \\
$B 3$ & $512 \times 512 \times 4096$ & 400 & 2.5 & 0.1 & 10 \\
$B 4$ & $512 \times 512 \times 4096$ & 600 & 2.5 & 0.1 & 15 \\
$B 5$ & $512 \times 512 \times 4096$ & 800 & 2.5 & 0.1 & 25 \\
\hline
\end{tabular}

$500 \times 500 \times 4000$ elements. This is beyond current computer capacities.

Thus we decided to use a cubic simulation domain (at $512^{3}$ elements) and to construct a bar-like domain by stacking this cube 8 times, each time rotating it and shifting it by one fifth of the domain size. Thus we get different realizations of the turbulent structures, mimicking a turbulent medium over the whole length of the line of sight.

\subsection{Model description}

Our "observational" domain is derived from a periodic-boxsimulation of the turbulent magnetized ISM by Li et al. (2000, 2004) and Heitsch et al. (2001), employing ZEUS-MP, the massively parallel version of ZEUS-3D (Norman 2000). The electron density and the magnetic field are rescaled to the values for the warm ISM, from which five models with different path lengths through the medium were constructed, as shown in Table 1.

The density and the magnetic field are initially uniform, and will be perturbed by a fixed velocity field. We generate the velocity field as described by Mac Low (1999), as follows. Each wave number $k$ is given an amplitude in Fourier space, which is randomly drawn from a Gaussian distribution around unity, and a random phase. Fourier transforming to velocity space yields velocity components in three dimensions for each position. This is the velocity field with which the model is perturbed at each timestep, to simulate turbulent driving. The velocity components are multiplied by a velocity amplitude at each timestep, chosen such that the input energy stays constant with time. Perturbations are only induced at the lowest spatial wavenumbers $1<k<2$. In the plane of sky, this corresponds to the largest scales possible in the domain. The driving mechanism is meant to mimic energy input on the largest scales by an unspecified physical process such as supernova shock fronts or Galactic shear.

We use a snapshot of the simulation at a time when the full turbulent cascade has developed and a steady state between energy input on the largest scales and numerical dissipation on the smallest scales has been reached. This steady state corresponds to a Mach number of $\mathcal{M} \approx 10$. This is more than the values usually assumed for the warm ISM (namely $\mathcal{M} \approx 1$ at a 
sound speed of $\approx 6 \mathrm{~km} \mathrm{~s}^{-1}$ ), thus rendering the turbulence more compressive than intended for this work.

However, the turbulent cascade represented by a Kolmogorov slope of $-5 / 3$ does not change substantially (see e.g., Cho et al. 2003), so that we can rescale the densities and field strengths to values appropriate for the warm ISM. Fluctuations in electron density are allowed up to an upper limit of $10 \%$ of the mean density (assumed to be $0.1 \mathrm{~cm}^{-3}$ ), which corresponds to the upper limit to structure in emission measure $(E M)$ in the field of observation given by highresolution $\mathrm{H} \alpha$ observations (Madsen, private communication). The field variations are of the order of the mean field of $2.5 \mu \mathrm{G}$.

We assume a filling factor of ionized gas of $100 \%$, and a sharp cutoff of Faraday rotation of the polarized emission at $600 \mathrm{pc}$. As the $R M$ is an integral over the line of sight, a smaller filling factor combined with a longer path length would give the same results.

The MHD model does not contain synchrotron emission within the medium itself. This is obviously not a realistic situation, but allows us to investigate the influence of beam depolarization without depth depolarization, as the latter only occurs in a medium that both Faraday rotates and emits. Including synchrotron emission in the medium would result in additional depolarization and larger deviations from the linear $\phi\left(\lambda^{2}\right)$ relation.

\subsection{Simulating beam depolarization}

The goal is to produce maps of Stokes $Q$ and $U$ from the models, which then can be treated like observational data. From the bars we construct the numerical $R M$ in the plane of sky as

$R M_{i, j}=0.81 \frac{L_{\mathrm{los}}}{N_{z}} \sum_{k=1}^{N_{z}}\left[n_{\mathrm{e}} B_{\|}\right]_{i, j, k}, \quad i, j \in\left\{1 \cdots N_{x}\right\}$,

in units of $\mathrm{rad} \mathrm{m}^{-2}$. The line-of-sight length $L_{\mathrm{los}}$ is given in parsecs, the electron density $n_{\mathrm{e}}$ in $\mathrm{cm}^{-3}$, and the magnetic field component along the line-of-sight $B_{\|}$in $\mu \mathrm{G} . N_{x}, N_{z}$ are the number of cells in $x$ and $z$ direction, respectively, and $N_{y}=N_{x}$. For simplicity, we integrate along parallel lines of sight instead of an integration over a cone-like domain. Stokes $Q_{0}$ and $U_{0}$ are derived from those maps via

$$
\begin{aligned}
& \phi=R M \lambda^{2}, \\
& U_{0}=\sin (2 \phi), \\
& Q_{0}=\cos (2 \phi) .
\end{aligned}
$$

Note that we assume $100 \%$ uniform background polarization, $P_{0}=\sqrt{Q_{0}^{2}+U_{0}^{2}}=1$, and a constant background polarization angle of $\phi_{0}=0$. The wavelength $\lambda$ is given in meters.

To mimic the limited telescope resolution, we smooth the Stokes $Q_{0}$ and $U_{0}$ maps with a Gaussian beam of width $\sigma$. This results in maps of $Q$ and $U$ which are $\sigma$ times oversampled and which now can be used like observational maps. A noise level of $0.05 Q, U_{\mathrm{rms}}$ is added to the Stokes parameters, which corresponds to the noise in the high signal-to-noise regions in the observations. $P$ and $\phi$ are calculated according to Eqs. (1) and (2). From the angle maps corresponding to the five observational wavelengths we finally can determine $R M_{\text {fit }}$ as

$\phi=R M_{\mathrm{fit}} \lambda^{2}$

We apply the same criteria to select reliable fits as in the observations (see end of Sect. 2).

Figure 3 gives an example for model $B 4$ at smoothing $\sigma=4$. $P$ shows elongated regions of depolarization, not directly related to features in the $R M$ map. However, the regions of low polarized intensity $P$ occur at locations of large gradients in $R M$. These structures reappear in the fitted $R M$, since regions of low $P$ are noise-dominated and thus do not allow an accurate fit. The other models show similar characteristics: elongated depolarized regions which are one beam wide are present in all models, and the $R M$ derived from smoothed data is patchy and shows sub-beam scale anomalies. However, a larger width of the $R M$ distribution $\triangle R M$ induces more depolarization, so that larger depolarized regions exist in those models. For further distinction between models, see Sect. 5 .

\section{Depolarization canals}

All high-resolution observations of the polarized intensity of the diffuse synchrotron background exhibit elongated, onebeam-wide "canals" of very low or zero $P$, where the radiation is depolarized (e.g., Duncan et al. 1997, 1999; Gray et al. 1999; Uyanıker et al. 1999; Gaensler et al. 2001; Haverkorn et al. 2003a,b). The polarization angle always changes by $90^{\circ}$ across such a canal (Haverkorn et al. 2000). Two possible explanations exist for the depolarization canals (see e.g., Burn 1966; Sokoloff et al. 1998):

\section{1. beam depolarization: if the gradient in} $R M \nabla R M=(2 n+1) \pi / 2$

within one beam, the radiation within the beam is depolarized. This mechanism would explain the fact that canals do not shift in position with frequency. However, it requires a medium with steep gradients in $R M$ and/or intrinsic angle $\phi_{0}$ within one beam, elongated perpendicularly to the gradient;

2. depth depolarization: a uniform synchrotron emitting magneto-ionic medium can cause complete depolarization for $|R M|=n \pi$. However, in this explanation the depolarization canals shift in position with frequency, and a uniform medium (across a major part of the path length) is needed for complete depolarization.

Haverkorn et al. (2004b) argue that in their observations (one of which is used here), beam depolarization due to $R M$ gradients is the dominant mechanism causing depolarization canals. This does not in any way mean that depth depolarization is not important in the rest of the medium. For observations made at $350 \mathrm{MHz}$, only a modest gradient in $R M$ across a beam $\left(\sim 2.1 \mathrm{rad} \mathrm{m}^{-2}\right)$ can create a canal, whereas much larger gradients $\left(\sim 34 \mathrm{rad} \mathrm{m}^{-2}\right)$ are needed to form a canal in $1.4 \mathrm{GHz}$ observations. For an interpretation of canals at $1.4 \mathrm{GHz}$ in terms of depth depolarization, see Shukurov \& Berkhuijsen (2003). 


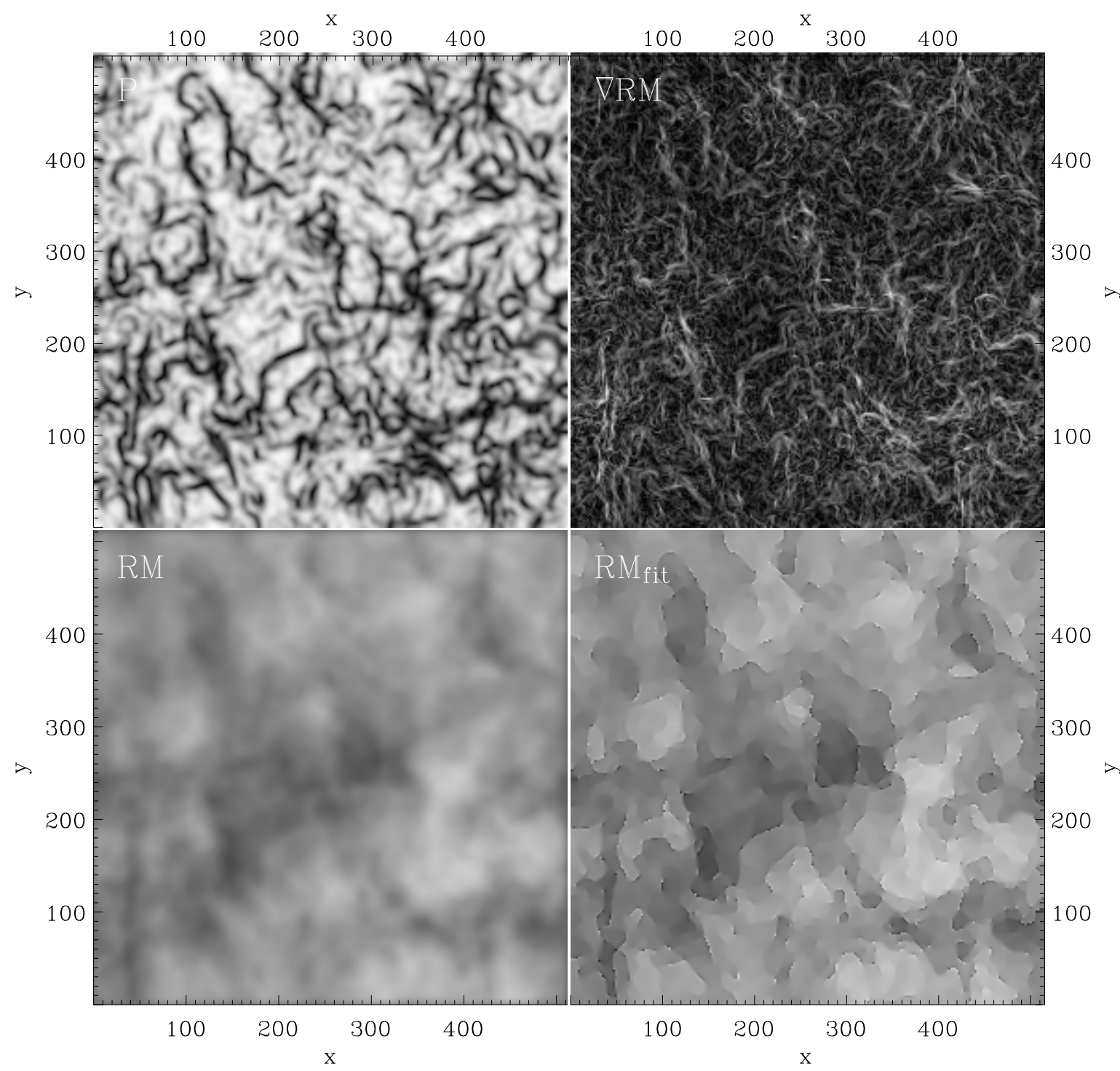

Fig. 3. Polarized intensity $P\left(4 \times 10^{-3}<P<0.97\right)$, gradient of $R M,|\nabla R M|\left(1 \times 10^{-3}<|\nabla R M|<4.9 \mathrm{rad} \mathrm{m}^{-2}\right.$ pixel $\left.^{-1}\right)$ and numerical and fitted $R M$ (scaled identically between $-6.8<R M<22.32 \mathrm{rad} \mathrm{m}^{-2}$ ) for model $B 4$ at smoothing $\sigma=4$, four times oversampled. Note that depolarized (black) canals appear at the locations of large (white) gradients. These regions reoccur in anomalous $R M_{\text {fit }}$, leading to fringes in the fitted $R M$ map.

In the numerical models discussed in Sect. 3, depolarization canals are visible as well (see top left map in Fig. 3), which are caused by beam depolarization.

At first sight, there is no obvious connection between the locations of the canals and $R M$ (left hand maps in Fig. 3). However, the magnitude of the gradient in $R M$ (top right map in Fig. 3) is obviously correlated to the position of the canals. This is demonstrated more quantitatively in Fig. 4, which shows a scatter plot of $P$ against the magnitude of the gradient in $R M$ with smoothing $\sigma=4$ for each independent "beam". The solid line denotes the theoretically expected depolarization in a beam due to a resolved gradient $\mathrm{d} R M / \mathrm{d} r$ in $R M$ (Sokoloff et al. 1998)

$$
\frac{P}{P_{0}}=\exp \left[-\frac{1}{\ln 2}\left(\frac{\mathrm{d} R M}{\mathrm{~d} r}\right)^{2} \lambda^{4}\right]
$$

where $P_{0}$ is the polarized intensity for constant $R M$ within the beam. The polarized intensity decreases almost to zero where the gradient in $R M$ is such that $\Delta \phi \approx \pi / 2$.

We selected "canal-like beams" by comparing $P$ in a certain point to the average $P$ of two diametrically opposed neighboring beams. If $P$ in the central beam was less than $20 \%$ of the averaged $P$ of the neighboring beams, the beam was considered a canal. Note that this selection criterion does not use any information about the length of the canals. Figure 5 shows the distribution of the gradient in $R M$ only at canals, defined according to the above process. The five plots show canals defined in five wavelengths, and the solid and dotted lines represent smoothings $\sigma=4$ and 8 , respectively. The dashed lines denote the value of $\nabla R M$ at the location where $\Delta \phi=-\pi / 2, \pi / 2$. Clearly, the gradient in $R M$ is peaked at the value where $\Delta \phi=\pi / 2$. The decline in number of canal pixels with frequency can be explained by the fact that the width 


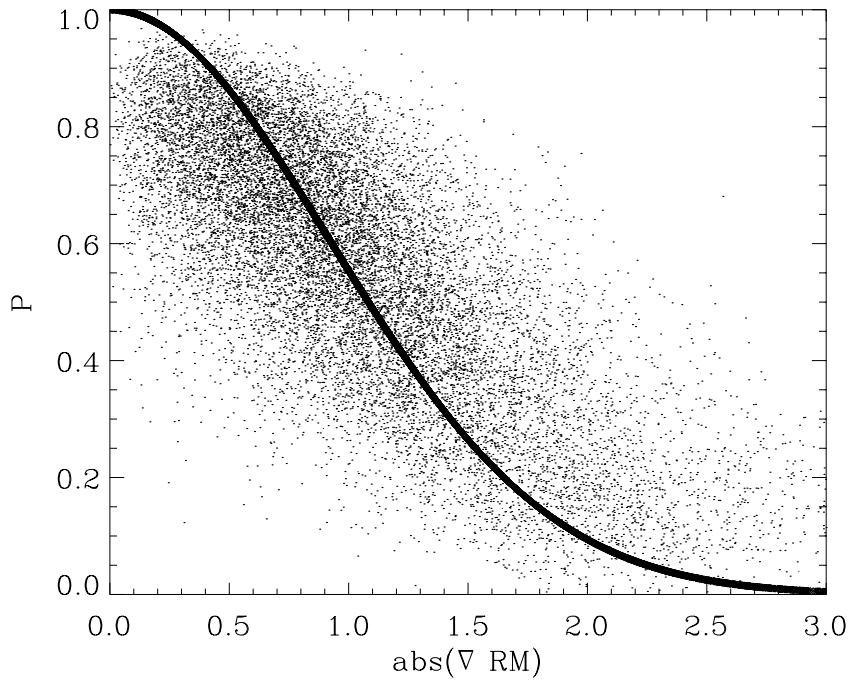

Fig. 4. Absolute value of the gradient in original $R M$ in the simulations $|\nabla R M|$ against polarized intensity $P$ of the smoothed maps with smoothing $\sigma=4$ for each pixel. The curve corresponds to Eq. (8).

of the polarization angle distribution decreases with increasing frequency for a given $R M$ distribution.

So the gradient of $R M$ in the canals has the right magnitude to cause beam depolarization (Fig. 5). Since the canals exist over many beams, the most likely direction of this gradient is perpendicular to the direction of the canals. In Fig. 6 we show $P$ in grey scale, with vectors of the $R M$ gradient superimposed, demonstrating that indeed the gradients in $R M$ are directed perpendicular to the canals.

Detailed analysis of the shape, direction and origin of $R M$ gradients and the implications on the medium will be discussed in a forthcoming paper. However, we give a simple estimate here of the required changes in magnetic field strength $B$ and/or electron density $n_{\mathrm{e}}$ to produce large enough gradients. For $\lambda=0.80 \mathrm{~m}, \Delta R M=2.45 \mathrm{rad} \mathrm{m}^{-2}$ in order to get an angle change of $\Delta \phi=\pi / 2$ within a beam. If the structures in $\nabla R M$ are filaments at an average distance of $300 \mathrm{pc}$, their width would be $0.4 \mathrm{pc}$. The necessary $\triangle R M$ requires an enhancement of $n_{\mathrm{e}}$ and $B$ of a factor 3 to 4 , for ISM parameters as in e.g. model $B 2$ (see Table 1). On the other hand, if the structures are sheetlike, and have an extent along the line of sight of a few parsecs, then an increase in electron density and magnetic field of $50 \%$ is sufficient, or an increase in either $n_{\mathrm{e}}$ or $B$ of $100 \%$. However, the unpublished $\mathrm{H} \alpha$ measurements discussed above (Madsen, private communication) give an upper limit to small-scale structure in emission measure $E M=\int n_{\mathrm{e}}^{2} \mathrm{~d} l \approx 0.3 \mathrm{~cm}^{-6} \mathrm{pc}$, which corresponds to a maximum $\Delta n_{\mathrm{e}} \approx 0.02 \mathrm{~cm}^{-3}$. Therefore, the gradients in $R M$ are probably mainly caused by magnetic field changes, and could originate from shocks, local narrow magnetic field reversals or other sheet-like structures in density and/or magnetic field.

Note that the canals in the numerical models are much shorter and less ordered than the ones in the observations. This is because in the numerical models we can introduce dynamical structure self-consistently only up to the domain size. Ideally, we would like to use subframes from the numerical models to be able to include larger scale structure in the models, so that modeling of canals across a significant part of the field would be possible. However, the available resolution is too low for this to be feasible.

Elongated narrow gradients in magnetic field and/or density have also been seen in other numerical simulations (e.g., Schekochihin et al. 2002; Cho et al. 2002).

Thus, in the numerical models discussed here, narrow and elongated gradients in $R M$ exist of large enough magnitude to cause beam depolarization in the modeled medium. Taking into account other observational evidence (Haverkorn et al. 2004b), this leads us to conclude that the narrow and deep depolarization canals as seen in the observations are most likely caused by beam depolarization due to $R M$ gradients.

\section{Reliability of $R M$ determinations}

The beam depolarization raises the issue of how reliable the $R M$ determinations are. Figure 7 quantifies the effect of limited resolution on the values of the computed $R M$. We determine the normalized mean quadratic difference between fitted and original smoothed $R M$ as

$\delta R M=\left(\frac{\left\langle\left(R M_{\sigma}-R M_{\mathrm{fit}}\right)^{2}\right\rangle}{\left\langle R M^{2}\right\rangle}\right)^{1 / 2}$,

where $R M$ is the original $R M$ and $R M_{\sigma}$ is $R M$ smoothed over Gaussian beams of width $\sigma$. We have chosen $\delta R M$ defined by Eq. (9) for the following reason. The goal of the equation is to compare the fitted $R M$ to the "real" $R M$ in the modeled medium. On scales below the beam size, the fitted $R M$ by definition cannot show any structure that represents structure in the medium. Therefore, if $R M_{\text {fit }}$ would be compared to the $R M$ as directly derived in the model, $R M_{\mathrm{fit}}-R M$ would give large values which are not the result of the smoothing, but only due to the fact that the beam size is incorporated in the determination of $R M_{\mathrm{fit}}$, but not in that of $R M$. Therefore, we chose to compare $R M_{\text {fit }}$ to an $R M$ which is smoothed over a Gaussian beam, assuming that the smoothed $R M$ approximates the physical $R M$ in the model if viewed only on scales larger than the beam size. In addition, the subsequent normalizing to the distribution width of the original unsmoothed $R M$ rules out any spurious effects on the difference estimates due to averaging. In this way, $\delta R M$ is a measure of how accurately the $R M$ computed from $Q$ and $U$ data with a finite beam width approximates the physical $R M$ in the medium.

Figure 7 shows the deviations $\delta R M$ for all independent beams satisfying the criteria for good fits (see end of Sect. 2). For a width of the $R M$ distribution $\Delta R M \approx 20 \mathrm{rad} \mathrm{m}^{-2}$ and a smoothing of $\sigma=4$ (values which are typical for the observations by Haverkorn et al. 2003a,b) we would expect from Fig. 7 an error of $\sim 20 \%$ in the fitted $R M$. However, as we will see below, this error estimate is an upper limit.

For $\Delta R M$ small enough not to lead to sign changes of $Q$ and $U$ within a beam, the differences $\delta R M$ saturate at a $5 \%$-level with increasing beam width, since variations of $R M$ within a beam are limited (Fig. 7 for $\triangle R M=3$ ). This behavior changes profoundly if Stokes $Q$ and $U$ reverse sign 


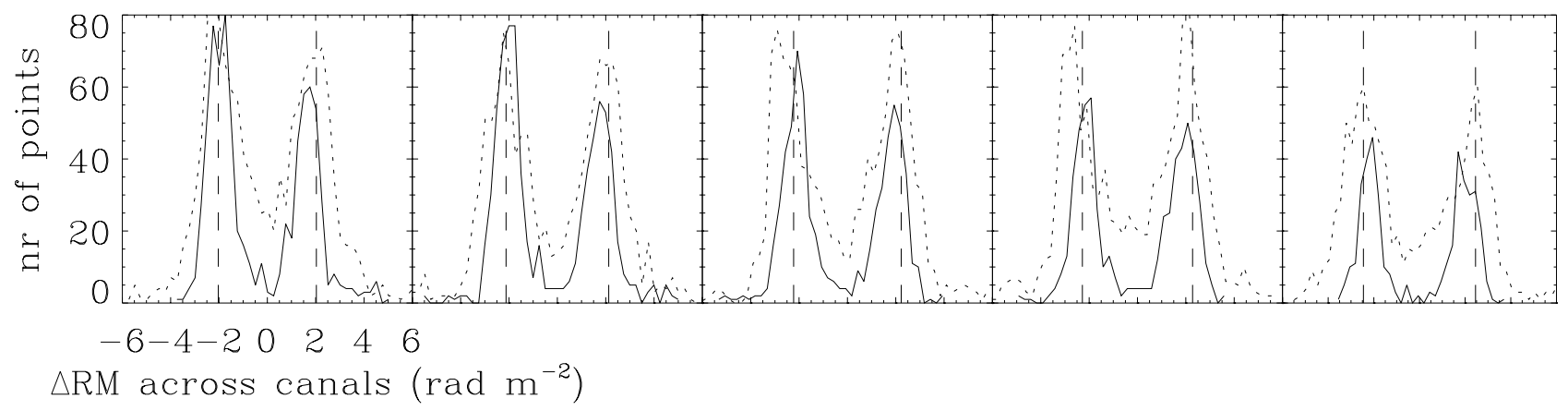

Fig. 5. Distribution of difference in $R M$ across a canal, $\Delta R M$. From left to right, canals are selected in wavelengths of $88 \mathrm{~cm}, 86 \mathrm{~cm}, 84 \mathrm{~cm}$, $83 \mathrm{~cm}$ and $80 \mathrm{~cm}$, respectively. The solid line denotes canals in $P$ maps smoothed by $\sigma=4$, the dotted lines are for a smoothing $\sigma=8$. The dashed lines give the positions where $\Delta R M \lambda^{2}=-\pi / 2, \pi / 2$.

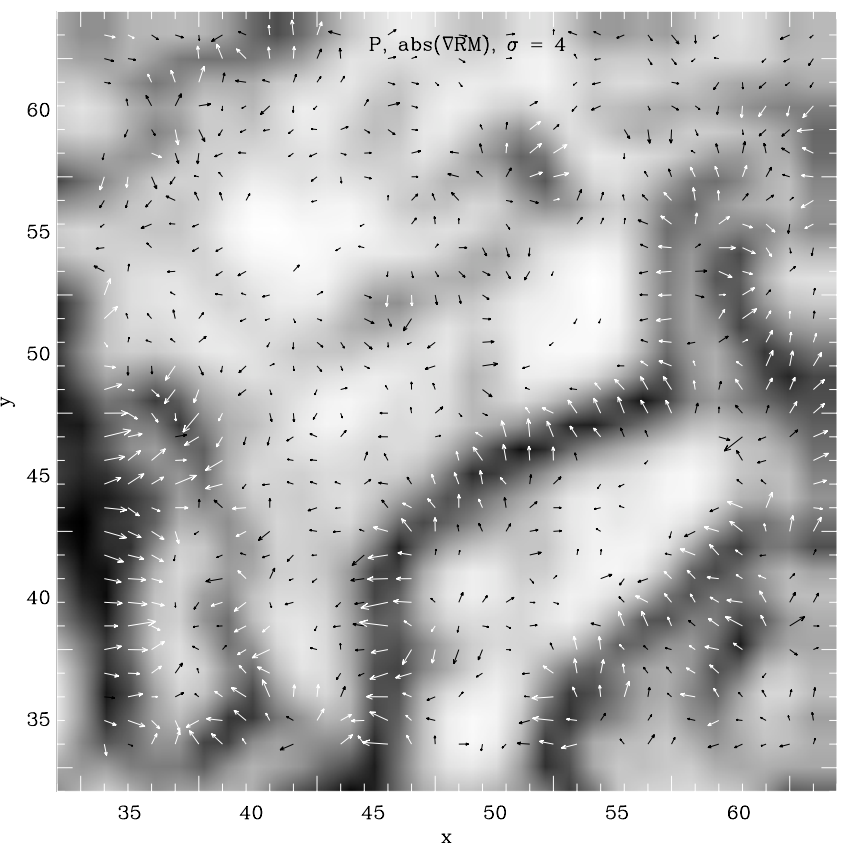

Fig. 6. Part of the polarized intensity map at smoothing $\sigma=4$ in grey scale, with strength and direction of the gradient in $R M$ superimposed as vectors.

within one beam. This is equivalent to a change in polarization angle of $90^{\circ}$ which, as discussed above, leads to depolarization canals. The effect on $\delta R M$ is twofold:

1. Low $P$ means small $S / N$, thus not well defined angles, and thus large errors in the fits. Therefore, those fits are excluded by the selection criterion.

2. Averaging introduces slight non-linearities in the angles around depolarized regions, i.e. regions where Stokes $Q$ and $U$ reverse sign within one beam. These non-linearities lead to an overshoot of the $R M$ (see Fig. 8) close to the depolarized region.

With increasing $\triangle R M$, Stokes $Q$ and $U$ are more likely to change sign within a beam. Beam averaging then leads to much severer depolarization (see Fig. 8). Depolarized regions are accompanied by systematic overshoots in $R M_{\text {fit }}$ (Fig. 8, top panel), which stem from the non-linearities in Eq. (2) arising when Stokes $Q$ changes sign. (Note that in the bottom panel

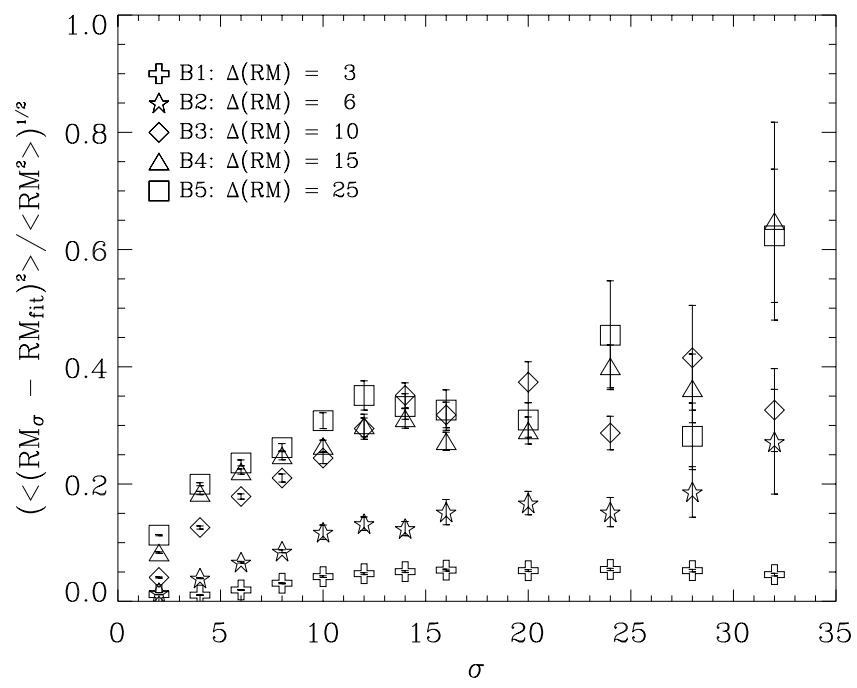

Fig. 7. Deviation of $R M, \delta R M$, calculated from smoothed $Q$ and $U$ maps from original $R M$, relative to original $R M$, against smoothing $\sigma$ (see Eq. (9)). Different symbols denote results for models $B 1$ to $B 5$ with a given width of the original $R M$ distribution. Only independent beam positions with $(P>5 S / N) \wedge\left(\chi^{2}<2\right)$ are used to determine $\delta R M$.

of Fig. 8, the polarization angles derived from beam-averaged Stokes $Q$ and $U$ are not ordered with respect to $\lambda$ any more in a canal, thus leading to ill-defined fits.) The overshoots of extreme positive and negative $R M$ are also visible in the observational data (see Fig. 2), indicating that the depolarization canals in the data are also caused by beam depolarization as opposed to depth depolarization.

Not all $R M$ gradients introduce these spurious results. Far away from the gradient, the fits retrieve the original $R M$. However, close to the gradient, multiple oscillations of $Q_{0}$ and $U_{0}$ within the gradient lead to $Q$ and $U$ close to zero over an extended region.

For one isolated $R M$-gradient as shown in Fig. 8a (see figure caption), the differences $\delta R M$ selected for reliable fits behave with the change in $R M$ across the canal $\triangle R M$ as shown in Fig. 9. The first spike corresponds to a 0th order canal with an angle difference of $\pi / 2$. Higher-order canals have angle differences of $(2 n+1) \pi / 2$, as discussed above. The $R M$ field from 


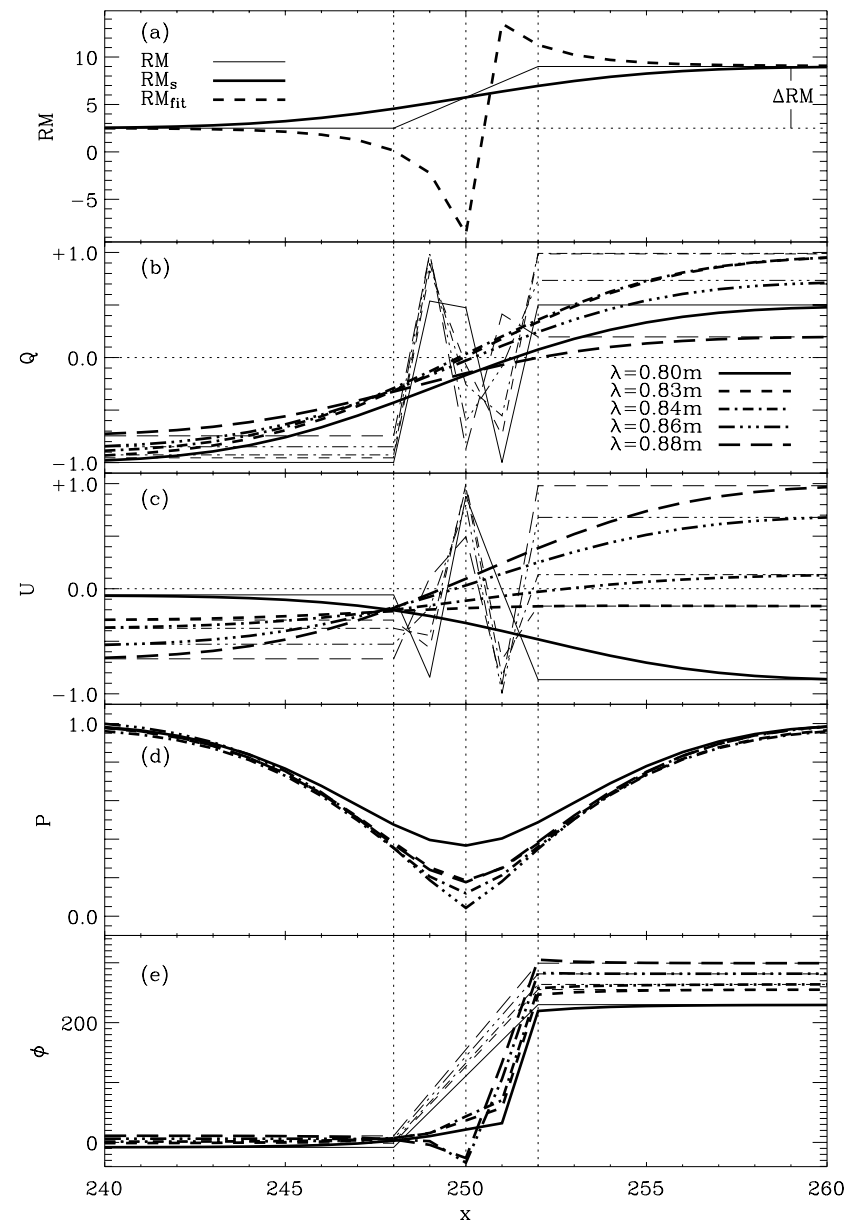

Fig. 8. Details of an idealized canal caused by sign changes in Stokes $Q$ (see text). a) $R M$ against spatial coordinate $x$. The original $R M$ (thin line) has a gradient between the two vertical dotted lines, leading to a smoothed $R M_{\mathrm{s}}$ (thick solid line) and a fitted $R M_{\mathrm{fit}}$ (thick dashed line) showing an overshoot, as in the observations. Smoothing ("beam") width corresponds to $\Delta x=4 . \Delta R M$ as used in the text is the change of $R M$ over the gradient, in this case $\Delta R M=6.5$. b) -d) stokes $Q, U$ and polarized intensity $P$ for all wavelengths before smoothing (thin lines) and after smoothing (thick lines): strong oscillations of the unsmoothed $Q$ and $U$ within the gradient region of $R M$ (see Eqs. (5) and (6)) lead to cancellation of smoothed $Q$ and $U$ within that region. Correspondingly, $P$ drops to nearly 0 . e) polarization angles for all wavelengths (as denoted in legend of $Q$ ). Thin lines show the angles derived from the original, unsmoothed $R M$, thick lines denote the angles derived from the smoothed $Q$ and $U$. Note that within the gradient region, there is no ordering of $\phi$ with $\lambda$.

the numerical models is a combination of all $\Delta R M \mathrm{~s}$ given in Fig. 9.

Note that the fitted $R M_{\text {fit }}$ deviates from the original $R M$ most for those $R M$ gradients where canals occur. In between the spikes, at positions where canals are not near, the difference $\delta R M$ drops nearly to zero, i.e. a finite beam width has almost no effect on the $R M$ determination. Therefore, $\delta R M$ is dominated by values around canals where $P>5 S / N$ (needed to be selected as reliable $R M$ ).

From Fig. 9 we conclude that the difference estimates $\delta R M$ for $\Delta R M>3 \mathrm{rad} \mathrm{m}^{-2}$ as given in Fig. 7 are indeed too pessimistic, since they are dominated by the regions around canals,

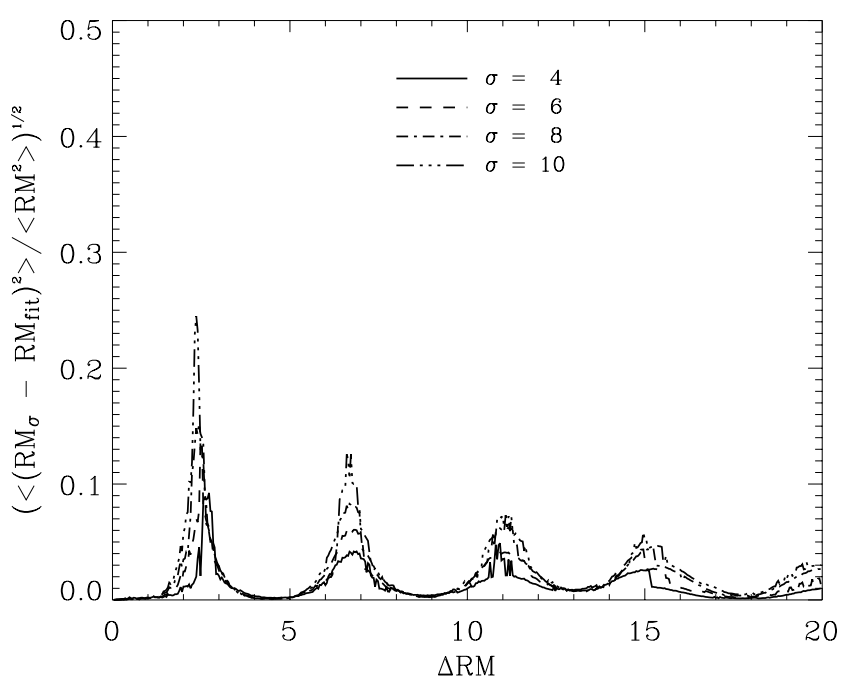

Fig. 9. $\delta R M$ (see Eq. (9)) for an isolated $R M$-gradient as shown in Fig. 8a against $\triangle R M$, the change of $R M$ across the gradient. The gradient width is 4 pixels while the beam width varies between 4 and 10 pixels. Fitted $R M$ s are derived in the same way as for the models, selected for $(P>5 S / N) \wedge\left(\chi^{2}<2\right)$ and independent beams. Note the periodicity $T_{R M} \approx 4.4$ in $\delta R M$, corresponding to an angle change of $\pi$. The first spike occurs at an angle difference of $\pi / 2$, the canal of 0 th order.

that are influenced by the canals but still fall within the definition of "reliable $R M \mathrm{~s}$ ". For smooth regions, or regions not affected by canals, we expect the $R M \mathrm{~s}$ determined from the observations to be accurate within $5 \%$ of $\triangle R M$, corresponding to an error of $0.15 \mathrm{rad} \mathrm{m}^{-2}$ for the observations mentioned here. The overall error will increase with increasing number of canals, which is why Fig. 7 generally shows larger $\delta R M$ than Fig. 9.

\section{Conclusions}

Multi-frequency radio observations of the Galactic synchrotron background allow us to probe the structure of the magnetoionized component of the ISM from $R M$ maps. However, the quality of the $R M$ determinations depends strongly on the degree of local depolarization due to intrinsic structure in the ISM, and thus in the $R M$. We investigated the effect of beam depolarization on the quality of the fitted $R M \mathrm{~s}$ with the help of numerical models. We find:

1. The models show depolarization canals due to beam depolarization across sharp gradients in $R M$. This suggests that narrow gradients of a few $\mathrm{rad} \mathrm{m}^{-2}$ can be a common feature in a magneto-ionized medium. Combined with other observational evidence, we therefore conclude that the depolarization canals seen in our $350 \mathrm{MHz}$ observations are caused by beam depolarization due to $R M$ gradients. While the width of the canals is an observational artifact, their presence and length mirror underlying structure in the Faradayrotating medium.

2. $R M$ determinations close to canals can be incorrect by several $\mathrm{rad} \mathrm{m}^{-2}$, depending on the absolute $R M$ difference between both edges of the canal. This is a consequence of a non-linearity in $\phi\left(\lambda^{2}\right)$ introduced by finite beam width. 
3. $R M$ determinations in smooth regions or in regions separated from canals by at least a beam are accurate to $0.05 \Delta R M$, where $\Delta R M$ is the width of the $R M$ distribution, or $\delta R M=0.15 \mathrm{rad} \mathrm{m}^{-2}$ for the observations mentioned here.

In future research, improved models will enable us to investigate what structures in the ISM actually cause the elongated gradients of $R M$, and will serve as a calibration what information about structure we can extract from observational maps.

Acknowledgements. The authors wish to thank Ellen Zweibel, Peter Katgert, Mordecai-Mark Mac Low and Enrique Vázquez-Semadeni for enlightening and invigorating discussions. We are grateful to P. S. Li and M. L. Norman for making the $512^{3}$ available, and thank Wolfgang Reich for useful and critical comments. Computations were performed on the SGI Origin 2000 of the National Center for Supercomputing Applications (NCSA) at Urbana-Champain. The Westerbork Synthesis Radio Telescope is operated by the Netherlands Foundation for Research in Astronomy (ASTRON) with financial support from the Netherlands Organization for Scientific Research (NWO). The project originated during the Astrophysical Turbulence Program at the KITP at University of Californa, Santa Barbara in 2000 (NSF grant PHY94-07194). M.H. is supported by NWO grant 614-21-006. F.H. is supported by a Feodor-Lynen fellowship of the Alexander-von-Humboldt Foundation and by NSF grant AST-0098701.

\section{References}

Armstrong, J. W., Rickett, B. J., \& Spangler, S. R. 1995, ApJ, 443, 209

de Avillez, M. A., \& Mac Low, M.-M. 2002, ApJ, 581, 1047

Burn, B. J. 1966, MNRAS, 133, 67

Cho, J., Lazarian, A., \& Vishniac, E. T. 2002, ApJ, 564, 291
Cho, J., Lazarian, A., \& Vishniac, E. T. 2003, ApJ, 595, 812

Crutcher, R. M. 1999, ApJ, 520, 706

Duncan, A. R., Haynes, R. F., Jones, K. L., \& Stewart, R. T. 1997, MNRAS, 291, 279

Duncan, A. R., Reich, P., Reich, W., \& Fürst, E. 1999, A\&A, 350, 447

Gaensler, B. M., Dickey, J. M., McClure-Griffiths, N. M., et al. 2001, ApJ, 549, 959

Gray, A. D., Landecker, T. L., Dewdney, P. E., et al. 1999, ApJ, 514, 221

Haverkorn, M., Katgert, P., \& de Bruyn, A. G. 2000, A\&A, 356, L1

Haverkorn, M., Katgert, P., \& de Bruyn, A. G. 2003a, A\&A, 403, 1031

Haverkorn, M., Katgert, P., \& de Bruyn, A. G. 2003b, A\&A, 404, 233

Haverkorn, M., Katgert, P., \& de Bruyn, A. G. 2004a, A\&A, submitted

Haverkorn, M., Katgert, P., \& de Bruyn, A. G. 2004b, A\&A, submitted

Heitsch, F., Zweibel, E. G., Mac Low, M.-M., Li, P. S., \& Norman, M. L. 2001, ApJ, 561, 800

Larson, R. B. 1981, MNRAS, 194, 809

Li, P. S., Norman, M. L., Heitsch, F., \& Mac Low, M.-M. 2000, BAAS, 197,502

Li, P. S., Norman, M. L., Mac Low, M.-M., \& Heitsch, F. 2004, ApJ, accepted

Mac Low, M.-M. 1999, ApJ, 524, 169

Mac Low, M.-M., \& Klessen, R. 2003, ARA\&A, in press [arXiv:astro-ph/0301093]

Minter, A., \& Balser, D. S. 1998, LNP, 506, 543

Norman, M. L. 2000, Rev. Mex. Astron. Astrofis. Conf. Ser., 9, 66

Reynolds, R. J. 1991, ApJ, 372, 17L

Schekochihin, A. A., Maron, J. L., Cowley, S. C., \& McWilliams, J. C. 2002, ApJ, 576, 806

Sellwood, J. A., \& Balbus, S. A. 1999, ApJ, 511, 660

Shukurov, A., \& Berkhuijsen, E. M. 2003, MNRAS, 342, 496

Sokoloff, D. D., Bykov, A. A., Shukurov, A., et al. 1998, MNRAS, 299, 189

Uyanıker, B., Fürst, E., Reich, W., Reich, P., \& Wielebinski, R. 1999, A\&AS, 138, 31 